\title{
Álgebra vs. Aritmética. Una propuesta didáctica que posibilita la construcción problematizada de un espacio matemático de trabajo constructivista en el aula
}

\author{
Algebra vs Arithmetic. A didactic proposal that allows the \\ problematized construction of a mathematical space of \\ constructivist work in the classroom
}

\section{Eugenio Ariel Valiero ${ }^{1}$}

\begin{abstract}
Resumen: Actualmente (finales de la segunda década del siglo XXI), se observa que, en las propuestas didácticas de las prácticas docentes de enseñanza secundaria, se ha desplazado la actividad aritmética escolar para dar mayor exclusividad a la enseñanza matemática a través del Álgebra. Este proceso se ha descripto como algebrización de la educación matemática escolar, que incluso se extiende más allá de la propia actividad de la Aritmética, y abarca también la didáctica de la Geometría, del Análisis Matemático, y de la Estadística.

El siguiente trabajo constituye un aporte reflexivo acerca de posibles propuestas didácticas que prescindan de la tarea con generalizaciones procedimentales, o algoritmos, y del uso de diferentes lenguajes y representaciones correspondientes al Álgebra. Para superar la situación descripta que enfatiza la enseñanza algebraica, se sugiere abordar la Aritmética en el contexto áulico de producción de saberes significativos (vinculado esto al constructivismo) en trabajo de colaboración entre pares estudiantes. En él, se abordarán dos propuestas: una efectivamente puesta en práctica con un grupo de 5 estudiantes de $2^{\circ}$ año de educación secundaria, y la otra, como sugerencia a desarrollar.
\end{abstract}

Fecha de recepción: 22 de junio de 2018. Fecha de aceptación: 06 de noviembre de 2019.

${ }^{1}$ Especialista Docente de Nivel Superior en Enseñanza de la Matemática en la Educación Secundaria. Ministerio de Educación de la Nación-Instituto Nacional de Formación Docente (MEN-INFoD). Profesor del Instituto de Enseñanza Superior "Dr. Luis Federico Leloir". Estudiante de la Maestría en Didáctica de las Ciencias/Matemática, Universidad Nacional del Rosario (UNR), Argentina. eugeniovaliero@gmail.com. orcid.org/0000-0001-9392-0882 
Palabras clave: Álgebra. Algebrización. Enseñanza de la Aritmética. Educación secundaria. Propuesta didáctica.

\begin{abstract}
At the moment (end of the second decade of the 21st century), it is observed that, in the didactic proposals of teaching practices of secondary education, the school arithmetic activity has been displaced to give greater exclusivity to the mathematical teaching through the Algebra. This process has been described as algebrization of school mathematics education, which even extends beyond the activity of Arithmetic itself, and also covers the didactics of Geometry, Mathematical Analysis, and Statistics.

The following work constitutes a reflexive contribution about possible didactic proposals that do without the work with procedural generalizations, or algorithms, and of the use of different languages and representations corresponding to the Algebra. To overcome the described situation that emphasizes algebraic teaching, it is suggested to approach Arithmetic in the aulic context of production of significant knowledge (linked to constructivism) in collaborative work between student peers. In it, two proposals will be addressed: one effectively implemented with a group of 5 students in the second year of secondary education, and the other, as a suggestion to develop.
\end{abstract}

Keywords: Algebra. Algebrization Teaching of Arithmetic Secondary education Didactic proposal.

\title{
INTRODUCCIÓN
}

Diferentes autores, entre ellos Irma Saíz, y Silvia Etchegaray (2016), señalan que la formación en competencias matemáticas, en los distintos niveles de los sistemas educativos, se ha centrado a lo largo de los años en el denominado proceso de algebrización, según el cual se construye una versión escolar de la matemática, basada en la producción de mecanismos de pensamiento y acción, donde se abordan con un enfoque desde el Álgebra las estructuras y propiedades de objetos matemáticos a ser enseñados. La situación no solo se expresa respecto de la didáctica del Álgebra, sino que se plantea también respecto de la enseñanza de la Geometría, del Análisis Matemático, y de la Estadística (Saiz, y Etchegaray, 2016). 
En tal sentido, puede admitirse, desde una mirada constructivista, que la algebrización del quehacer aritmético en el aula, se vería enriquecida en caso en que se priorizaran niveles de abordaje que comprendan las enseñanzas y los aprendizajes.

Godino, Neto, Wilhelmi, Aké, Etchegaray y, Lasa (2015), describen 3 niveles de razonamiento algebraico, basados en distinciones ontosemióticas: presencia de objetos algebraicos intensivos, tratamiento de dichos objetos mediante operaciones y transformaciones con propiedades de estructuras algebraicas, y la presencia de distintos tipos de lenguajes ya sean natural, icónico, gestual o simbólico (Godino, Neto, Wilhelmi, Aké, Etchegaray, y Lasa, 2015).

Visto de esa manera, todo proceso de algebrización de la Aritmética, incluye dichos niveles al enseñar contenidos aritméticos. Desde esa perspectiva, en las aulas de educación secundaria se encontrarían propuestas didácticas que vincularan la enseñanza de la Aritmética a pautas de resolución de problemas mediante algoritmos convencionales, referidos a relaciones entre operaciones y a tipologías que se incorporarían a la actividad matemática como modelos prácticos mecanicistas e incuestionables; lo propio, en ese marco, es que tales propiedades se acepten sin demostración, o que se den secuencias de resolución estandarizadas, o generalizaciones técnicas como expresión de la algebrización, en lugar de trabajarse en el aspecto numérico.

Es decir, que los formatos de representación y operación, los distintos lenguajes, los principios, las relaciones o propiedades aritméticas que representan situaciones o modelizan un problema, por lo general, son dados, externos a la actividad de resolver, no se construyen en el aula para el caso que se resuelve, sino que son creados con anterioridad y, extemporáneos al proceso no solo de aprendizaje sino también al de enseñanza; están allí desde antes que existiera o se presentara a los estudiantes el problema, listos para ser usados, para dar respuesta a intereses matemáticos preexistentes.

Dicho panorama, conforma un marco didáctico de referencia donde lo que se aprende deja fuera la posibilidad creativa de producir las propias referencias y afirmaciones para contextualizar lo que los profesores enseñan y lo que los estudiantes aprenden. Se establece así un entorno de fundamentación técnica, lo cual significa que el conocimiento aritmético se valida mediante un conjunto de normas de operación, por procedimientos pautados con cierta anticipación a la enseñanza y al aprendizaje.

Esto ocurre muchas veces cuando las propiedades aritméticas son transmitidas en forma acrítica y a-reflexiva. Un ejemplo lo constituye la aceptación de 
la muy difundida, en la formación escolar, propiedad que indica que "Ios números cuya última cifra es 0 o 5, son divisibles por 5". Este criterio, más allá de su validez general, se enseña con carácter acrítico, incuestionable, y pareciera dar cuenta de una propiedad a la cual en el aula solo nos limitamos a corroborar cada vez. Esta modalidad de trabajo y admisión del conocimiento se podría atribuir más que a un sistema matemático, a un sistema de creencias escolares que se aceptan con carácter técnico y de saber operativo.

De acuerdo a lo que señalan los investigadores Cortés, Hitt, y Saboya (2016), es necesario generar espacios de trabajo matemático escolar que fortalezcan el pensamiento aritmético-algebraico. En ese sentido, caracterizan al pensamiento aritmético como aquel que tiene que ver con el concepto y sentido asignado a lo numérico, al significado de las operaciones, al control aritmético, al cálculo mental y a la lectura y escritura de problemas como habilidades aritméticas (Cortés, Hitt, y Saboya, 2016).

Los mismos autores, citando a Kaput $(2000,2008)$, mencionan que: (...) el pensamiento algebraico debe comportar en general dos aspectos principales: 1 . Realizar y expresar la generalización de los sistemas simbólicos de manera más formal y convencional, y 2. Razonar con formas simbólicas, incluyendo la manipulación sintáctica guiada de estas formas simbólicas (Cortés, Hitt, y Saboya, 2016). Debe señalarse además que, las investigaciones de Cortés, Hitt, y Saboya, se dirigen no a pregonar la enseñanza de la Aritmética separada de la del Álgebra, como aseguran se ha venido haciendo durante muchos años para recortar el currículum escolar con contenidos para educación primaria y secundaria, respectivamente, sino a sugerir una didáctica holística entre ambas, desde los primeros años de escolarización (Cortés, Hitt, y Saboya, 2016).

Otros investigadores, tales como Tarriga, y Sierra (2014), han desarrollado propuestas metodológicas de enseñanza del Álgebra para pasar de lo concreto a lo abstracto, en procesos mediados por materiales manipulables, y materiales de tecnología digital (Tarriga, y Sierra, 2014).

No obstante, se observa que las prácticas en el aula han radicalizado la tendencia a sostener el conocimiento matemático a partir de generalizaciones más del lado del Álgebra, exagerando la capacidad de abstracción como vía de conocimiento, lo cual disminuye la significatividad en la clase de Matemática del pensamiento aritmético, y olvidando que el verdadero problema cognitivo debería radicar en generar preguntas acerca de si son siempre válidas ciertas reglas, en plantearse la necesidad de conocer cómo se obtienen las mismas, en poder decidir en qué segmento del campo numérico son aplicables o verdaderas, 
en abocarse a la búsqueda de los criterios utilizables para argumentar su validez, y en generar un entorno de enseñanza y de aprendizaje problematizado.

\section{LA PROBLEMATIZACIÓN COMO ESTRATEGIA ESCOLAR}

En contraposición a lo dicho, es dable pensar, entonces, en propiciar un contexto de producción del quehacer matemático problematizado en la escuela, lo cual demandaría, según Patricia Sadovsky y Carmen Sessa² (2005), centrar la acción en los procesos y actividades matemáticos posibilitados en entornos de colaboración grupal; es decir, fomentar la participación en comunidades matemáticas, basando sus recomendaciones en la denominada Teoría de las Situaciones Didácticas, de Brousseau, desde donde se admite que el aprendizaje matemático se construye en vínculo con los contextos culturales y sociales. (Sadovsky y Sessa, 2005).

La actividad matemática a la que se refieren las autoras remite a un proceso donde es poco habitual la incorporación explicitada y consciente de saberes didácticos acerca de distintas complejidades cognitivas, por la que, en interacción con otros, se adquieren habilidades para: conjeturar, argumentar, ejemplificar, contra-ejemplificar, contrastar, validar, generalizar, establecer condiciones, definir, simbolizar, representar, contextualizar, descontextualizar, ajustar, justificar, analizar posibilidades, demostrar. Todo ello, a partir del trabajo matemático que debe sostenerse, y retroalimentar en doble sentido el pensamiento propio de cada estudiante y el de sus pares en trabajo colaborativo. Es decir, se desconocen los ordenamientos, las etapas, los momentos y las lógicas de secuenciación de mecanismos, que hacen que dichas habilidades evolucionen desde lo más simple a lo más complejo.

\section{PROPUESTA DIDÁCTICA REAL DE TRABAJO ARITMÉTICO EN EL AULA}

Interesaba, al poner en práctica esta propuesta, conocer qué ocurriría respecto del trabajo aritmético en contexto escolar mediante actividades de resolución de problemas; observar los tipos de interacciones entre pares de estudiantes, y las

2 Docentes e investigadoras del grupo a cargo del Postítulo destinado a Docentes de Educación Primaria SEGCBA. 
elaboraciones de trabajo matemático grupal. Para ello se propuso a un grupo de 5 estudiantes de $2^{\circ}$ año de la Escuela Secundaria, una situación a resolver formada por 3 consignas, entregadas de a una a la vez, en formato escrito, y leídas en voz alta:

(a) Para dirigirse a un aeropuerto, un contingente de 96 turistas se moviliza de la siguiente manera. Por cada turista que viaja en un taxi, 5 viajan en un autobús. ¿Cuántos turistas utilizan cada medio de transporte?

(b) Si la relación ahora fuera siempre de 4 a 6, ¿qué cantidad de turistas podrían formar el contingente, movilizándose de esa manera?

(c) Encontrar una propiedad aritmética que permita caracterizar todas las cantidades posibles de turistas que puede tener un contingente conservando siempre la relación de 4 a 6. (Saiz y Etchegaray, 2016).

Los estudiantes fueron seleccionados al azar, de un grupo escolar urbano de 28 alumnos cuyos rendimientos académicos, en promedio, no presentaban particularidades sobresalientes, es decir que los mismos no eran alumnos con los mejores rendimientos de aprendizajes, pero tampoco presentaban problemáticas al respecto. Debe aclararse, además, que la elección no se realizó teniendo en cuenta afinidades personales o frecuencia de agrupamiento entre pares en las tareas de aulas; y que, la escuela donde se aplicó la secuencia es una institución pública que pertenece a un barrio céntrico de la ciudad de Gualeguay, provincia de Entre Ríos (Argentina), que promueve como modalidad de enseñanza y de aprendizaje en trabajo en grupos o equipos de estudiantes.

Los alumnos aceptaron rápidamente participar de la actividad para lo cual se les comunicó que se retirarían del aula y que resolvería en un aula apartada sin la presencia del resto de los compañeros o de otros estudiantes de la escuela, y fuera de la clase de Aritmética. También se les aclaró que no constituía una evaluación, que no serían calificados, y que podían disponer de los elementos que comúnmente utilizaban en la clase, por lo que llevaron consigo los útiles tales como hojas, lápices, y calculadora. Se les dijo además que podían conversar entre ellos, y registrar lo que fueran necesitando a medida que resolvían, y que dispondrían de no más de 80 minutos. El docente aplicador de la secuencia fue un profesor de Matemática, estudiante de un curso de especialización superior en enseñanza de la Matemática para la educación secundaria, distinto al docente habitual del grupo seleccionado, que procuró intervenir solo para satisfacer consultas y hacer aclaraciones no procedimentales. 
Mediante la primera consigna, se esperaba que los estudiantes abordaran la pregunta y construyeran un modelo aritmético propio respecto de las posibilidades de agrupar a 96 viajantes en taxi o autobús, de acuerdo a las condiciones dadas y mediante la interacción entre pares. Era posible que los estudiantes tomaran el problema como un simple caso de divisibilidad, o que se cuestionaran respecto de los agrupamientos que se debían hacer, y llegaran a advertir que debían construir criterios de solución basados en más de una posibilidad. Respecto del trabajo disciplinar se preveía una tendencia a resolver preconcibiendo que habría una fórmula para ello, dado que es esa una característica de la algebrización.

La segunda consigna, sugería producir cambios en las condiciones que acentuaran la perspectiva no algebrista. En ambas se ponía en juego también la importancia de concebir respuestas no únicas, en enriquecer la participación de todos los integrantes del grupo con el aporte de cada uno, y en analizar lo que ocurriría respecto de la validez de lo que fueran afirmando respecto de la/s respuesta/s a la pregunta. Se buscaba, además, que los estudiantes se cuestionaran si se podría tratar de afirmaciones para un caso, o para unos casos particulares, o para todos los casos, si se efectuaran nuevas modificaciones. La última consigna (c), explicitaba dicho cuestionamiento, a la vez que diera cuenta de las habilidades de algebrización por parte de los estudiantes.

\section{ANÁLISIS DE DESARROLLO DE LA PROPUESTA}

Ante la consigna (a), los estudiantes inicialmente demoraron en organizar su trabajo, debiendo leer varias veces, y formularon sus primeras anotaciones de maneras difusas y desordenadas. En una etapa inicial no se consultaron entre ellos, pero rápidamente la mayoría hizo intentos de escribir múltiplos de 5. Luego de leer varias veces la consigna en forma individual, algunos de ellos se abocaron inmediatamente a localizar una expresión o fórmula que colaborara con la resolución de la consigna, y escribieron datos relacionados con el número de turistas (96), y simbolizaciones referidas a las cantidades mínimas de pasajeros y a la clase de transporte (1T.....5A). Luego de unos minutos algunos iniciaron la construcción de una tabla que vinculaba al número 1 con el 5, al 2 con el 10, al 3 con el 15. Esta tarea ya fue más abierta a la participación colectiva. El entusiasmo hizo que ese proceso se comunicara entre los estudiantes y en poco tiempo construyeran una tabla completa que resolvió la situación: 


\begin{tabular}{|c|c|c|}
\hline 1 & 5 & 6 \\
\hline 2 & 10 & 12 \\
\hline 3 & 15 & 18 \\
\hline 4 & 20 & 24 \\
\hline 5 & 25 & 30 \\
\hline$\ldots$ & $\ldots$ & $\ldots$ \\
\hline$\ldots$ & $\ldots$ & $\ldots$ \\
\hline 16 & 80 & 96 \\
\hline
\end{tabular}

Colectivamente, mediante la participación de tres estudiantes, propusieron la regularidad advertida por la que se completaban las dos primeras columnas. La tercera columna, correspondiente a la suma de los elementos vinculados en las dos primeras, fue agregada y completada posteriormente, siendo advertida la importancia informativa de su agregado, por uno solo de los estudiantes. Los mismos acordaron como válido el procedimiento y se produjo un momento de mayor confianza en la propuesta, observaciones, y agregados individuales para dar respuesta a la actividad planteada.

En tal sentido, Cambriglia, Sadovsky, y Sessa (2010), proponen pensar que la interacción entre docentes y alumnos, y entre alumnos, propicia la aparición de inquietudes, puntos de vista, acepciones personales, y posicionamientos que establecen el ámbito de construcción de las generalizaciones (Cambriglia, Sadovsky, y Sessa, 2010).

Por otra parte, si bien el registro tabular validó el procedimiento, no pudo precisarse un enunciado general que completara la generalización, no al menos explícitamente, puesto que sí se establecieron generalizaciones; una de ellas es la que asigna a cada grupo de pasajeros la suma entre 1 y 5, que, a la vez genera la secuencia de la tercera columna, donde la "ley" es la suma de 6 en 6 .

Siguiendo lo expresado por las mismas autoras mencionadas anteriormente, es posible identificar rasgos históricos institucionales, respecto de la matemática escolar, en las intenciones iniciales, de estos alumnos, de encontrar una fórmula para que rápidamente diera la solución. Esto se podría vincular a las experiencias de aprendizaje matemático vividas con anterioridad en cada trayectoria estudiantil. También, se podría asociar a la afirmación que se hiciera unos párrafos atrás, por la cual la formación académica en la escuela secundaria refiere a conocimientos técnicos, que solicitan del último nivel de abstracción matemática, como lo es el de algebrización, en desmedro de los procesos de 
aritmetización, donde se pretende un abordaje numérico, de sus relaciones y de las propiedades de las operaciones.

De todas formas, el modelo aritmético (tabla, sucesiones numéricas), donde se prioriza el control de los significados del contexto numérico (número de pasajeros, cantidades, situación problemática, sumas), prevaleció por sobre la intención de elaborar un modelo algebraico por parte de algunos estudiantes. Esto se hizo más evidente en el momento en el que encontraron un criterio de regularidad de construcción de la tercera columna. Pudo advertirse, además, que los estudiantes tuvieron gran participación en la producción y desglose de la información que requería la solución, constituyéndose en integrantes productores de conocimiento contextualizado a la situación apropiada por el grupo.

Algunos aspectos a tener en cuenta son los que se asocian a cuestiones de vocabulario y lenguaje. Los estudiantes consultaron al profesor el significado de algunas palabras presentes en las consignas, por ejemplo, el de "contingente", e incluso dijeron desconocer el término "aritmético", pero no manifestaron inquietudes respecto de lenguajes algebraicos o acerca de registros de representación. Debe mencionarse además que el momento de la construcción de la estrategia de resolución contó con instantes de comunicación entre estudiantes donde se proponían "saltear pasos", "sumar todo", "sumar los grupos de 5", "contar hasta llegar a...". La actividad exploratoria más destacada se generó en los momentos iniciales donde se produjo el diálogo entre pares con pruebas y anotaciones de multiplicaciones para aproximarse a 96.

No obstante, no se presentaron elementos discursivos claros de formulación de conjeturas por parte de los estudiantes (excepto la tabla donde se sistematizó el procedimiento particularizado en cada uno de los casos); es decir, que no manifestaron en lenguaje coloquial verbalmente o por escrito, otras formas de formulaciones de enunciados y afirmaciones verificables o contrastables.

De acuerdo a lo anteriormente dicho, lo deseable hubiera sido que, como afirman María Elena Markiewicz, y Silvia Etchegaray (2006), hubieran surgido conjeturas nutridas, las cuales, según las autoras, constituyen Primitivas desde donde las mismas, y sus consecuencias generales, se hacen contrastables frente a conjeturas menores, y así se sostienen o se derriban. Esto puede deberse a lo que anteriormente se dijo respecto de la cultura matemática escolar, que da mayor importancia a los procesos algebraicos, y en menor medida a la construcción de un razonamiento de lo plausible. Al respecto, las investigadoras nos dicen: 
En el contexto escolar, en particular en la escuela media, no hay demasiados espacios que permitan el desarrollo del razonamiento plausible, la reflexión acerca del mismo y la toma de conciencia de su papel y de su importancia dentro de la matemática.

Esto genera en el ámbito de la clase de matemática, una suerte de "confusión argumentativa" producto de la falta de distinción entre distintas formas de validación. (Markiewicz, y Etchegaray, 2006).

Respecto de la consigna (b), pudieron rápidamente darle solución, en tanto que el procedimiento utilizado fue muy similar, sin necesidad de escribir todos los pares relacionados. Los estudiantes indicaron a partir de dos multiplicaciones $(9 x 4, y$ 9x6), que el número de turistas sería 90. La tabla que exhibieron fue la de los 3 primeros valores, y se mostraron sorprendidos de saber que "sobran 6 turistas".

\begin{tabular}{|c|c|c|}
\hline 4 & 6 & 10 \\
\hline 8 & 12 & 20 \\
\hline 12 & 18 & 30 \\
\hline
\end{tabular}

Si bien advirtieron que la cantidad de turistas sería múltiplo de 10, la respuesta a la consigna (c), se dirigió a encontrar la relación entre los valores de la segunda columna, con los de la primera:

$$
6 T / 4 A=3 / 2 \quad 12 T / 8 A=3 / 2 \quad 18 T / 12 A=3 / 2
$$

Esto hizo que la suposición obtenida al principio no fuera validada, sino que arribaran a un resultado no apropiado donde la respuesta dependía de condiciones que no explicitaron. Se expone así que la solución a un problema o situación aritmética depende en gran medida de la contextualización numérica, y que, si bien iniciaron un proceso de control sintáctico de la situación modelizada, la algebrización provocó dificultades o inconsistencias por no ser aplicable siempre el resultado obtenido a todos los casos. Esto último no fue advertido ni cuestionado por parte de los estudiantes. La manera de dar solución a la última consigna muestra que en dicho momento se llegó a un cierto margen mayor de algebrización que desde el principio fue en aumento, pero que no logra establecerse concretamente en el tercer nivel del que hablan Godino, Neto, Wilhelmi, Aké, Etchegaray, y Lasa (2015), puesto que no se construyeron distintos 
tipos de lenguaje y representaciones que dieran una variedad representativa, comunicacional u ontosemiótica.

Verónica Cambriglia (citada anteriormente), habla también, parafraseando a Mercier (1998), acerca de cierta inversión de roles en el aula, en donde por momentos la acción del alumno se parece a la del profesor, y viceversa. Esos espacios de intercambio no se observaron en la propuesta de la actividad; no más que en forma de oposición ante la inseguridad que presentaron los estudiantes frente a cálculos de algunas multiplicaciones mientras intentaban responder la consigna (b). Uno de los estudiantes dudó de haber multiplicado bien, y en ese momento otro estudiante expresó que ese era el valor correcto: $-9 \times 6$ es 54... ¿sí?... - Sí. hay que repasar las tablas!

\section{LA DIVISIBILIDAD COMO POSIBLE PROBLEMA DE CONSTRUCCIÓN DE SABER ALGEBRAICO}

Otra actividad como ejemplo para poner en acto el trabajo aritmético de producción que se sugiere en el aula podría ser la que se vincula a la divisibilidad (Teorema Fundamental de la Aritmética). Tómese el caso de los siguientes interrogantes, que se presentan sintéticamente, si haber sido puestos a prueba como actividad en un grupo particular de alumnos, pero que se propone desarrollar a partir de los siguientes planteamientos:

Interrogante 1

¿Saben cuántos divisores tienen $5^{2}$ y $5^{3}$ ? ¿Cuántos divisores tendrá $5^{n}$, siendo $n$ un número natural?

Interrogante 2

Un número natural tiene exactamente 8 divisores naturales, dos de ellos son 35 y

77 ¿Cuál es el número? (Instituto Nacional de Formación Docente, 2016).

A partir de cierta exploración numérica podría iniciarse el estudio de los divisores de las potencias segunda y tercera de 5 . Estos casos particulares solucionan parcialmente el Interrogante 1 , si se admite la divisibilidad de $5^{2}$ entre los únicos divisores naturales 1,5, y 25 , a los que se pueden incorporar los divisores en $Z$ $(-1,-5, y-25)$, es decir serían 3 divisores naturales y 6 divisores enteros. Para $5^{3}$, puede procederse de igual manera, por lo que se reconocen 4 divisores naturales 
y un total de 8 en el campo de los enteros. Una pregunta que podría surgir es si esos divisores son los únicos, y de qué manera puede confirmarse tal afirmación, o aseverarse. Tal conjetura no quedaría demostrada con un contraejemplo, pero es posible que los estudiantes hagan el intento si es que no poseen la certeza, de localizar otros divisores, sin encontrarlos.

Pero el procedimiento admite otras posibilidades, como lo es el de descomposición de la base de potencia en factores primos, de donde el número de divisores resulta de la suma de divisores primos y las combinaciones posibles entre ellos. Esta conjetura, también requerirá ponerse a prueba.

Se dirá entonces que el trabajo matemático en estos casos, es contextualizado en un problema aritmético, y cuando se desea generalizar los resultados, se hace necesario construir otras conjeturas que pueden obtenerse como extensión del trabajo anterior. Esto significa que el número de divisores para la potencia tercera, cuarta, y sucesivas, posiblemente responda a una regularidad conjeturable, de la que se debe obtener una validación antes de extender la solución a un número $\mathrm{n}$ en el exponente de la potencia de 5 .

Una alternativa de producción en el aula sería entonces continuar la identificación de los divisores para las "n" primeras potencias naturales:

51: $1 ; 5 ;-1 ;-5$. (2 divisores en $N^{3}$ y 4 divisores en $\left.Z^{4}\right)$

52 : $1 ; 5 ; 25 ;-1 ;-5 ;-25$. (3 divisores en $N$ y 6 divisores en Z)

$5^{3}: 1 ; 5 ; 25 ; 125 ;-1 ;-5 ;-25 ;-125$. (4 divisores en $\mathrm{N}$ y 8 divisores en Z)

54 : $1 ; 5 ; 25 ; 125 ; 625-1 ;-5 ;-25 ;-125 ;-625$. (5 divisores en $N$ y 10 divisores en $Z$ )

...

5n: $(n+1)$ en N y $(n+1) \times 2$ en Z. (conjetura solución).

Esta conjetura, que progresa desde una práctica aritmetizada a una construcción algebrizada, requerirá ser verificada, validada y generalizada. La solución así planteada requiere continuar la clarificación del ámbito de validez, y las condiciones bajo las cuales se cumplirá. Los estudiantes seguramente se inquietarían ante afirmaciones referidas a los divisores propuestos de cada potencia, pudiendo cuestionarlos e iniciar procesos de validación que faciliten mayor certeza, y riqueza de producción.

\footnotetext{
3 N: número natural.

4 Z: número entero.
} 
El Interrogante 2, requiere analizar lo que sucede con los divisores conocidos. Si n es el número buscado, y sabiendo que es divisible por $35=5 \times 7$, y por 77 $=7 \times 11$; luego es también divisible por 5, por 7 , y por 11 . Se sabe además que todo número es divisible por sí mismo y por la unidad, es decir que 1 también es divisor. Todos los productos de las combinaciones entre los divisores serán divisores. Esto significa que $5 \times 11=55$, lo es. El producto entre 5, 7, y 11 (385), también es divisor de dicho número n puesto que resulta divisible por 35, 55, y 77. Por ser 385 el mayor de los divisores que admite divisores a sí mismo y la unidad, entonces $n=385$; quedando el siguiente conjunto de divisores:

\begin{tabular}{|l|l|l|l|l|l|l|l|}
\hline 1 & 5 & 7 & 11 & 35 & 55 & 77 & 385 \\
\hline
\end{tabular}

Al igual que para el Interrogante 1, en este caso es necesario que se expliciten las condiciones en las que se cumpliría esta solución, por ejemplo que el enunciado haya solicitado los divisores naturales, los criterios de selección de los factores primos, y las afirmaciones que se fueron imponiendo en el desarrollo, por ejemplo, dar argumentos que validen la afirmación respecto de que, si 35 es divisor, los divisores de 35 son también divisores de n. Éstas, podrían presentarse como limitantes de la producción en clase, o como oportunidad para la formulación de nuevos interrogantes de conocimiento y expansión de propiedades aritméticas, de casos particulares a generales.

\section{CONCLUSIÓN}

La Aritmética podría constituirse en una herramienta que posibilite el hacer matemático a la vez que genere una actitud de enseñanza y de aprendizaje significativos, de manipulación y comunicación de objetos de conocimiento de la Matemática escolar, ya sean conceptos, teorías, definiciones, acepciones y condiciones especiales de construcción de saberes en un proceso de exteriorización de propiedades y de relaciones opuesto a la idea simple de "aplicación de técnicas dadas", de algoritmos para resolver, que vincule a las propuestas didácticas con la idea de problematización, es decir, que introduzca posibilidades de preguntarse, por parte de los estudiantes, y de elaborar caminos de hipotetización, validación, y verificación de saberes que en el trabajo matemático se pueden ir construyendo en la tarea de dar solución a un determinado problema aritmético. 
Es así que, construir en Matemática en el aula, y particularmente en Aritmética, abriría la posibilidad no solo de resolver significativamente una situación matemática planteada, un determinado problema, una pregunta con contenido aritmético, sino también, como intentó ponerse en práctica en la actividad propuesta, dar lugar a la emergencia de significados, conceptualizaciones, apreciaciones, y a la aplicación de contenidos teóricos aprendidos en la práctica con las consiguientes producciones y concepciones emergentes en la propia tarea de manipulación de objetos matemático por parte de los estudiantes.

En particular, en la actividad propuesta a los alumnos, la secuencia didáctica se presentó bajo la modalidad de resolución de problemas, pero son escasos los elementos que muestran verdaderas interacciones de un proceso altamente significativo entre las personas que intervinieron, ya sean docente y alumnos o alumnos entre sí en interacción de pares, como procesos comunicacionales propios de la modalidad de enseñanza y aprendizaje a través de problemas. También, son escasos los elementos observados que puedan dar cuenta del desarrollo de una tarea netamente constructivista de contenidos aritméticos, puesto que en un primer momento sí surgió la tendencia por parte de los estudiantes de ir en búsqueda de una formulación algebraica.

Construir, en tal sentido, implicaría aplicar saberes previos, y producir nuevos saberes que se ajusten a los requerimientos del trabajo matemático particular, es también un proceso que se construye, y no sin admitir que problematizar, en las enseñanza y en los aprendizajes aritméticos, es instalar interrogantes como práctica habitual, y generar las condiciones sociales de participación colectiva; debe ser parte del proceso natural de adquisición de propiedades, y del contexto matemático productivo en el que lo sabido se ponga en duda y deba ser sometido a pruebas en las actividades grupales entre pares.

\section{REFERENCIAS}

Cambriglia, V. Sadovsky, P. y Sessa, C. (2010). Procesos Colectivos de Generalización. Publicado en las Memorias de la III REPEM 2010. Santa Rosa, La Pampa. http://repem. exactas.unlpam.edu.ar/cdrepem10/memorias/comunicaciones/Trabajos\%20Inves/ CB\%2049.pdf 
Cortés, J. C.; Hitt, F; y Saboya, M. (2016). Pensamiento Aritmético-Algebraico a través de un Espacio de Trabajo Matemático en un Ambiente de Papel, Lápiz y Tecnología en la Escuela Secundaria. Bolema, 30 (54), 240-264. http://dx.doi.org/10.1590/1980-4415v30n54a12. http://www.scielo.br/scielo.php?pid=S0103-636X2016000100240¿script=sci_abstract\&tlng=es

Godino, J. D., Neto, T., Wilhelmi, M. R., Aké, L., Etchegaray, S., Lasa, A. (2015). Niveles de algebrización de las prácticas matemáticas escolares. Articulación de las perspectivas ontosemiótica y antropológica. Avances de Investigación en Educación Matemática, 8 , 117-142. http://funes.uniandes.edu.co/9249/

Instituto Nacional de Formación Docente. (2016). Clase 06. Continuamos trabajando con la relación de divisibilidad en N y en Z. Módulo Enseñanza de la Aritmética. Programa Nuestra Escuela. Formación Docente Continua. Especialización docente de Nivel Superior en Enseñanza de la Matemática en la Escuela Secundaria. Ministerio de Educación y Deportes de la Nación. Buenos Aires.

Markiewicz, M. E. y Etchegaray, S. C. (2006). Algunos Resultados de una Investigación Acerca del Razonamiento Plausible o Conjetural. Memorias de la I REPEM 2006. Santa Rosa, La Pampa. http://repem.exactas.unlpam.edu.ar/cdrepem06/memorias/ comunicaciones/Trabinvest/CTI2.pdf

Tarriaga, M; y Sierra, J. (2014). CONEXIONES ALGEBRAICAS: Metodología de enseñanza-aprendizaje del álgebra para pasar de lo concreto a lo abstracto con el apoyo de tecnología emergente. Congreso Iberoamericano de Ciencia, Tecnología, Innovación y Educación. 12, 13, y 14 de noviembre de 2014. Buenos Aires. Argentina.

Sadovsky, P., \& Sessa, C. (2005). La Conformación de una Comunidad Matemática en un Proceso de Formación de Maestros: un Ejemplo Privilegiado para Conocer Complejidades Acerca de la Clase de Matemática. Yupana, 1(2), 11-24. https://doi.org/10.14409/ yu.v1i2.242.

Saiz, I. y Etchegaray, S. (2016). Clase 01. Iniciación a un estudio didáctico-matemático de la Aritmética. Módulo Enseñanza de la Aritmética. Programa Nuestra Escuela. Formación Docente Continua. Especialización docente de Nivel Superior en Enseñanza de la Matemática en la Escuela Secundaria. Ministerio de Educación y Deportes de la Nación. Buenos Aires.

EUGeNIO ARIEL VALIERO

Dirección: Calle: Colón, Núm. 326, Código postal 2840

Ciudad Gualeguay, Entre Ríos, Argentina 